ABSTRACTS OF CONTRIBUTED PAPERS

\title{
EMISSIVITIES OF Fe III AND Fe VI AND IRON ABUNDANCES IN PLANETARY NEBULAE
}

\author{
R.H. Garstang and W. Derek Robb* \\ Joint Institute for Laboratory Astrophysics, \\ University of Colorado, Boulder \\ * (Now at Los Alamos Scientific Laboratory, New Mexico)
}

We have calculated electron impact excitation cross sections between most of the low metastable levels of the $3 \mathrm{~d}^{6}$ configuration in Fe III and of the $3 \mathrm{~d}^{3}$ configuration in Fe VI have also been computed. The statistical equilibrium equations have been solved for a range of electron temperatures and electron densities, and the resulting level populations used to calculate emissivities for the forbidden lines of $\mathrm{Fe}$ III and Fe VI. Comparisons with observed line intensities and abundance determinations in planetary nebulae are in progress. (Paper submitted to The Astrophysical Journal.)

\section{DISCUSSION}

Garstang: I would like to open the discussion on my own paper by asking the question many of you have been asking me: could I do [FeII] crosssections? The answer is yes, in principle. We hope to do [FeV] first and then [FeII], but it may be a while before we have the results because of limitations of human time and computer time. We also hope to do some photoionization cross-section calculations for various stages of ionization of iron.

Aller: Theoretical difficulties in obtaining collisional cross-sections for metastable levels in ions of iron arc liacched only by observational difficulties in measuring intensities of weak iron forbidden lines. They were first detected hv Bowen and Wyse (1938); Walker and I (1970) tried to measure -ines ( $\lambda 4600-\lambda 590 n)$ with the Lallemand electronic camera. Shortward of $\lambda 4700$ high dispersion Coudé observations were used to measure intensities of highly ionized iron. Since these lines are typically a thousand times weaker than $H \beta$, their intensities had to be related to prominent nebular lines via photographic rather than photoelectric measurements. By using the image tube scanner it is possible to extend the wavelength range and improve on the older measurements as well as re-calibrate the Lallemand tube data. 
Garstang: In those objects in which several iron lines have been seen the relative intensities seem to agree tolerably well with our calculated values. Even if you can only measure the strongest line with acceptable accuracy that would be valuable and probably sufficient for an ionic abundance determination.

Osterbrock: Higher stages of ionization, namely [FeX] $\lambda 6374$ and [FeXI] $\lambda 7892$ are clearly present in several Seyfert galaxies. It would be worthwhile to look carefully for these lines in high-ionization planetaries, particularly NGC 7027 because they furnish information on the input radiation field at very high energies.

Garstang: I agree, but one should notice that according to our simple calculations on ionic abundances of iron there is a wide range of ionizing radiation temperatures for which much of the iron is in the FeVIII and FeIX stages, so that a rather high temperature would be required to produce FeX.

Nussbaumer: Collision strengths and transition probabilities for the Fe ions can be calculated, but to what accuracy? We calculate at present FeVI data, the collision strengths by the Distorted Wave method. The differences between the results of the two calculations may give a hint about uncertainties associated with such calculations.

Garstang: There are many uncertainties in the calculations, and it is indeed difficult to make an assessment of what the uncertainties are. An independent calculation would help considerably in this respect. Improvements in the photoionization cross-sections are also of great importance in trying to improve iron abundance determinations.

THE BOWEN FLUORESCENCE MECHANISM FOR SPHERICALLY SYMMETRIC PLANETARY NEBULAE

R.Wehrse and A. Peraiah

Institut für Theoretische Astrophysik

Heidelberg, Germany

The transfer equation for the interlocked HeII Lya/OIII $2 \mathrm{p}^{2} \mathrm{P}_{2}$ $2 \mathrm{p} 3 \mathrm{~d} \mathrm{P}_{2}{ }^{\circ}$ lines is solved for spherical symmetry and for comparison in plane parallel approximation in the framework of the discrete space theory of radiative transfer. We used Harrington's ionization model (1969) without velocity fields and applied the partial redistribution function $\mathrm{R}_{\mathrm{IIA}}$ for both lines.

Appreciable differences occur between the two cases. In spherical symmetry the lines are narrow in the outer regions of the nebula, but much stronger than for plane parallel geometry so that more photons 